\title{
European Journal of Nuclear Medicine and Molecular Imaging
}

Direct prospective comparison of 18F- FDG PET and arterial spin labelling MR using simultaneous PET/MR in patients referred for differential diagnosis of dementia

\author{
--Manuscript Draft--
}

\begin{tabular}{|c|c|}
\hline Manuscript Number: & \\
\hline Full Title: & $\begin{array}{l}\text { Direct prospective comparison of } 18 \mathrm{~F}-\mathrm{FDG} \text { PET and arterial spin labelling MR using } \\
\text { simultaneous PET/MR in patients referred for differential diagnosis of dementia }\end{array}$ \\
\hline Article Type: & Original Article \\
\hline Keywords: & arterial spin labelling ASL MRI; 18F-FDG PET; dementia; PET/MR; brain imaging \\
\hline Corresponding Author: & $\begin{array}{l}\text { Jenny Ceccarini, PhD } \\
\text { University Hospitals Leuven and KU Leuven } \\
\text { Leuven, --- Please Select --- BELGIUM }\end{array}$ \\
\hline $\begin{array}{l}\text { Corresponding Author Secondary } \\
\text { Information: }\end{array}$ & \\
\hline Corresponding Author's Institution: & University Hospitals Leuven and KU Leuven \\
\hline $\begin{array}{l}\text { Corresponding Author's Secondary } \\
\text { Institution: }\end{array}$ & \\
\hline First Author: & Jenny Ceccarini, PhD \\
\hline First Author Secondary Information: & \\
\hline Order of Authors: & Jenny Ceccarini, PhD \\
\hline & Sophie Bourgeois, MD \\
\hline & Donatienne Van Weehaeghe, MD \\
\hline & Karolien Goffin, MD PhD \\
\hline & Rik Vandenberghe, MD PhD \\
\hline & Mathieu Vandenbulcke, MD PhD \\
\hline & Stefan Sunaert, MD PhD \\
\hline & Koen Van Laere, MD PhD \\
\hline Order of Authors Secondary Informe & \\
\hline Funding Information: & \\
\hline Abstract: & $\begin{array}{l}\text { Purpose: 18F-FDG PET is routinely used as imaging marker in the early and } \\
\text { differential diagnosis of dementing disorders and has incremental value over the } \\
\text { clinical neurological and neuropsychological evaluation. Perfusion imaging by means } \\
\text { of arterial spin labelling (ASL) is an alternative modality to indirectly measure neuronal } \\
\text { functioning and could be used as complement measurement in a single MR session in } \\
\text { the workup of dementia. Using simultaneous PET-MR, we performed a direct head-to- } \\
\text { head comparison between enhanced multiplane tagging ASL (eASL) and 18F-FDG } \\
\text { PET in a true clinical context of subjects referred for suspicion of neurodegenerative } \\
\text { dementia. } \\
\text { Methods: Twenty-seven patients underwent a 20-minute 18F-FDG PET/MR and } \\
\text { simultaneously acquired eASL on a GE Signa PET/MR. Data were compared to } 30 \\
\text { screened age- and gender-matched healthy controls. Both integral eASL and } 18 \text { F-FDG } \\
\text { datasets were analysed visually by two readers unaware of the final clinical diagnosis, } \\
\text { either in normal/abnormal classes, or full differential diagnosis (normal, Alzheimer type } \\
\text { dementia [AD], dementia with Lewy Bodies [LBD], frontotemporal dementia [FTD] or } \\
\text { other). Reader confidence was assessed in a qualitative four-point scale. Data were } \\
\text { also analysed semiquantitatively by VOI and voxel-based analyses. } \\
\text { Results: The ground truth diagnosis for the patient group resulted in } 14 \text { patients with a } \\
\text { neurodegenerative cognitive disorder (AD, FTD, LBD) and } 13 \text { patients with no } \\
\text { arguments for an underlying neurodegenerative cause. Visual analysis resulted in }\end{array}$ \\
\hline
\end{tabular}




\begin{tabular}{|l|l|}
\hline & $\begin{array}{l}\text { equal specificity (0.70) for differentiating normal and abnormal cases between the two } \\
\text { modalities, but in a higher sensitivity (0.93) and confidence rating for 18F-FDG PET } \\
\text { compared to eASL (0.64). The same was true for assigning a specific differential } \\
\text { diagnosis (18F-FDG PET: 0.61; eASL: 0.39). Semiquantitative analyses revealed } \\
\text { prototypical patterns for AD and FTD, with for both a higher effect size on 18F-FDG } \\
\text { PET. } \\
\text { Conclusion: In a direct head-to-head comparison on a simultaneous GE Signa } \\
\text { PET/MR, 18F-FDG PET performs better in term of sensitivity, reader confidence, effect } \\
\text { size and more intense abnormalities than ASL. However, using pure semiquantitative } \\
\text { analysis, similar diagnostic accuracy between the two modalities was obtained. } \\
\text { Therefore, ASL may still serve as complement to neuroreceptor or protein deposition } \\
\text { PET studies when a single simultaneous investigation is warranted. }\end{array}$ \\
\hline Suggested Reviewers: & $\begin{array}{l}\text { Alexander Drzezga } \\
\text { alexander.drzezga@uk-koeln.de }\end{array}$ \\
\hline Marion Smits \\
marion.smits@erasmusmc.nl
\end{tabular}




\title{
TITLE PAGE
}

Jenny Ceccarini $\mathrm{PhD}^{1^{*}}$, Sophie Bourgeois $\mathrm{MD}^{2 *}$, Donatienne Van Weehaeghe $\mathrm{MD}^{1,2}$, Karolien Goffin

MD $\mathrm{PhD}^{1,2}$, Rik Vandenberghe MD $\mathrm{PhD}^{3}$, Mathieu Vandenbulcke MD $\mathrm{PhD}^{4}$, Stefan Sunaert MD

$\mathrm{PhD}^{1,5}$, Koen Van Laere MD PhD DrSc ${ }^{1,2}$

*Shared first author

\section{Direct prospective comparison of ${ }^{18}$ F-FDG PET and arterial spin labelling MR using simultaneous PET/MR in patients referred for differential diagnosis of dementia}

\author{
Affiliations \\ Belgium. \\ 2. Division of Nuclear Medicine, University Hospitals Leuven, Leuven, Belgium. \\ 3. Department of Neurology, University Hospitals Leuven, Leuven, Belgium \\ 4. Old Age Psychiatry, University Hospitals Leuven, Leuven, Belgium \\ 5. Department of Radiology, University Hospitals Leuven, Leuven, Belgium.
}

1. Department of Imaging and Pathology, University Hospitals Leuven and KU Leuven, Leuven,

Corresponding author: Jenny Ceccarini, University Hospital Leuven, Herestraat 49, 3000 Leuven. jenny.ceccarini@uzleuven.be, Tel : +32 16343715

ORCID : 0000-0003-2774-9516

\section{Acknowledgements}

The authors thank all the participants for their willingness to participate in this study, the PET radiopharmacy, research technologists (Kwinten Porters and Jef Van Loock) and radiology team of UZ Leuven for their skilled support. Jenny Ceccarini and Donatienne Van Weehaeghe are postdoctoral and $\mathrm{PhD}$ fellows of the Research Foundation Flanders (FWO) respectively. Rik Vandenberghe and Koen Van Laere are Senior Clinical Investigators of the FWO. 


\section{ABSTRACT}

Purpose: ${ }^{18}$ F-FDG PET is routinely used as imaging marker in the early and differential diagnosis of dementing disorders and has incremental value over the clinical neurological and neuropsychological evaluation. Perfusion imaging by means of arterial spin labelling (ASL) is an alternative modality to indirectly measure neuronal functioning and could be used as complement measurement in a single MR session in the workup of dementia. Using simultaneous PET-MR, we performed a direct head-to-head comparison between enhanced multiplane tagging ASL (eASL) and ${ }^{18}$ F-FDG PET in a true clinical context of subjects referred for suspicion of neurodegenerative dementia.

Methods: Twenty-seven patients underwent a 20-minute ${ }^{18}$ F-FDG PET/MR and simultaneously acquired eASL on a GE Signa PET/MR. Data were compared to 30 screened age- and gender-matched healthy controls. Both integral eASL and ${ }^{18}$ F-FDG datasets were analysed visually by two readers unaware of the final clinical diagnosis, either in normal/abnormal classes, or full differential diagnosis (normal, Alzheimer type dementia [AD], dementia with Lewy Bodies [LBD], frontotemporal dementia [FTD] or other). Reader confidence was assessed in a qualitative four-point scale. Data were also analysed semiquantitatively by VOI and voxel-based analyses.

Results: The ground truth diagnosis for the patient group resulted in 14 patients with a neurodegenerative cognitive disorder (AD, FTD, LBD) and 13 patients with no arguments for an underlying neurodegenerative cause. Visual analysis resulted in equal specificity (0.70) for differentiating normal and abnormal cases between the two modalities, but in a higher sensitivity $(0.93)$ and confidence rating for ${ }^{18} \mathrm{~F}$-FDG PET compared to eASL (0.64). The same was true for assigning a specific differential diagnosis $\left({ }^{18}\right.$ F-FDG PET: 0.61; eASL: 0.39). Semiquantitative analyses revealed prototypical patterns for AD and FTD, with for both a higher effect size on ${ }^{18}$ F-FDG PET.

Conclusion: In a direct head-to-head comparison on a simultaneous GE Signa PET/MR, ${ }^{18}$ F-FDG PET performs better in term of sensitivity, reader confidence, effect size and more intense abnormalities than ASL. However, using pure semiquantitative analysis, similar diagnostic accuracy between the two modalities was obtained. Therefore, ASL may still serve as complement to neuroreceptor or protein deposition PET studies when a single simultaneous investigation is warranted. 
Key words: arterial spin labelling ASL MRI; ${ }^{18}$ F-FDG PET; dementia; PET/MR; brain imaging.

1

2

3

4

5

7

8

10

11

12

13

14

15

16

17

18

19

20

21

22

23

24

25

26

27

28

29

30

31

32

33

34

35

36

37

38

39

40

41

42

43

44

45

46

47

48

49

50

51

52

53

54

55

56

57

58

59

60

61

62

63

64

65 


\section{INTRODUCTION}

The prevalence of dementia is rapidly increasing. Based on the World Alzheimer's Report, almost 50 million people worldwide currently have dementia, and with aging population the current prognosis is that more than 130 million individuals will be afflicted worldwide by 2050 [1,2]. Several underlying disorders can cause dementia, but the four most common are Alzheimer's disease (AD), Lewy body dementia (LBD), vascular dementia (VD) and frontotemporal dementia (FTD). Extracellular amyloid plaques, along with neurofibrillary tangles, are a neuropathologic hallmark of $\mathrm{AD}$ [1]. The guidelines from the National Institute on Aging-Alzheimer's Association (NIA-AA) suggest a variety of ancillary biomarker tools to increase the clinical confidence in diagnosis of $\mathrm{AD}$, including cerebrospinal fluid biomarkers, MR volumetry, fluorodeoxyglucose $\left({ }^{18} \mathrm{~F}-\mathrm{FDG}\right)$, amyloid and tau PET [3]. Amyloid PET already has a major impact in management of patients suspected for $\mathrm{AD}$ [4] and is offered as standard of care in many institutions. Current research efforts address the role of tau PET in the workup of dementia [5-7]. However, ${ }^{18}$ F-FDG PET still remains the most frequently used, widely available and well-established functional imaging tool to assess neuronal functioning and to differentiate dementia patients with high diagnostic accuracy even early in the course of the disease $[3,8,9]$, with an average sensitivity of 0.91 and specificity of 0.85 for diagnosing AD. ${ }^{18} \mathrm{~F}-\mathrm{FDG}$ PET also is an established biomarker for distinguishing FTD and LBD from other dementias [10,11].

Arterial spin labelling (ASL) magnetic resonance imaging has been proposed as an alternative noninvasive, radiation free and practical alternative functional marker, based on the neurovascular coupling, and thus also a proxy marker for neuronal function [12]. Several authors have suggested that ASL could be an emerging biomarker for diagnosing $\mathrm{AD}$ and other neurodegenerative conditions [13-15]. ASL can be seen comparable to SPECT perfusion imaging [16] with respect to spatial resolution and has been shown to produce similar regional patterns of hypoperfusion in patients with various types of dementia [13]. Moreover, ASL uses magnetically labelled arterial blood water as an endogenous tracer and can be used to quantify cerebral blood flow (CBF) in an absolute way (ml/100 g brain/min).

Several imaging studies have reported good correlations between hypoperfusion as measured with ASL and hypometabolism using ${ }^{18}$ F-FDG PET, both in patients with AD or FTD [17-23], suggesting that 
ASL could be an alternative to ${ }^{18} \mathrm{~F}$-FDG PET in the diagnosis of dementia syndromes. However, the

diagnostic value of ASL in clinical dementia diagnosis on an individual basis has yet to be determined. The reported sensitivity and specificity of ASL (range sensitivity: 0.53-0.80; range specificity: 0.620.84) compared to ${ }^{18} \mathrm{~F}-\mathrm{FDG}$ PET largely varies among studies, probably due to differences in ASL techniques, type of comparative analysis and due to the heterogeneity of small cohorts [17-23].

So far, no direct comparison between ASL and ${ }^{18}$ F-FDG PET has been reported measured by means of simultaneous PET/MR. By using simultaneous imaging, potential day to day variations, medication effects or effect of evolutive comorbidities can be minimalized, enabling an optimal direct comparison. In this work, we have therefore compared the diagnostic accuracy of ${ }^{18} \mathrm{~F}$-FDG PET and pulsed enhanced multiplane tagging eASL using a simultaneous PET/MR system in the most challenging but clinically relevant setting of patients with suspected dementia, in comparison to carefully screened healthy controls. The aim of the study was to establish visual accuracy for blinded readers, as well as to assess semiquantitative volume-of-interest (VOI) and voxel-based analysis to classify patients as either abnormal ('neurodegenerative pattern') or not, as well as to perform a more elaborate differential diagnosis. 


\section{MATERIALS AND METHODS}

The study was approved by the Ethics Committee Research UZ / KU Leuven (Leuven) and written informed consent was obtained from all participants.

\section{Study population}

Twenty-seven consecutive patients (age $64.3 \pm 11.2$ years, $14 \mathrm{M} / 13 \mathrm{~F}$ ) were prospectively included between December 2016 and June 2017. All had been referred from the local tertiary memory clinic for a brain ${ }^{18}$ F-FDG PET scan because of recent cognitive decline and a question of potential neurodegenerative dementia. All patients underwent routine clinical, neurological and extensive neuropsychological examination in their workup, and in most cases also structural MRI (T1 and FLAIR) was performed. CSF A $\beta$ and tau data were available in 5 patients, no amyloid PET scan results were available at the time of ${ }^{18} \mathrm{~F}-\mathrm{FDG}$ PET/MR. The working diagnosis at the time of ${ }^{18} \mathrm{~F}-\mathrm{FDG}$ PET scan referral is shown in Table 1. As ground truth diagnosis, the last available diagnosis made by the memory clinic physician was taken, based on all routinely available information, and established after follow-up up to 1.5 years (Table 1$)$.

Thirty healthy controls (CON; age $63.9 \pm 10.6$ years; $14 \mathrm{M} / 16 \mathrm{~F})$ were recruited trough advertisements in local newspapers and on the departmental websites. The main exclusion criteria for this group included: history of neurological or psychiatric disorders, first degree relative with neurodegenerative dementia, important systemic pathologies (e.g. diabetes, cancer, liver or kidney disease) or use of any central acting medication. All controls underwent a neurological examination by a board-certified physician, had a mini-mental state (MMSE) score $\geq 28$, Beck Depression Inventory score $\leq 9$, and a normal T1 and T2 MRI for their age. The control subjects were part of a large ${ }^{18}$ F-FDG PET/MR normal aging database, of which a randomised age- and gender-matched subset was randomly selected for this study.

\section{Image acquisition}

All subjects fasted at least 4 hours prior to ${ }^{18} \mathrm{~F}-\mathrm{FDG}$ injection. For patients, intravenous tracer injection was performed under standardized circumstances (supine, low ambient light, low noise, eyes open). 
Patients first underwent clinical routine ${ }^{18} \mathrm{~F}-\mathrm{FDG}$ brain PET/CT, 30-min post-injection of $150.5 \pm 11.5$ unit and received a second 20 minute list-mode PET acquisition on a simultaneous 3 Tesla Signa PET/MR system (GE Healthcare, Chicago, IL, USA). Control subjects underwent a dynamic 60-min PET/MR scan started directly after intravenous injection of $152.2 \pm 11.1 \mathrm{MBq}{ }^{18} \mathrm{~F}-\mathrm{FDG}$ (range: 131 $185 \mathrm{MBq}$ ). The first 15 minutes of the simultaneous scan, no MR sequences were applied in order not to invoke primary auditory cortex activation and subjects were asked to keep eyes open. From the listmode data, the last 20-min were reconstructed (40-60 min p.i.) as static scan and used as comparator for this study.

Vendor-based MR-based attenuation correction (MRAC) corrected PET images were reconstructed using ordered subset expectation maximization (OSEM) with 6 iterations and 28 subsets, and postsmoothed with a $3 \mathrm{~mm}$ isotropic Gaussian filter. MR image acquisition was performed during the PET acquisition using an 8-channel high resolution head array coil (GE Healthcare). In addition to an anatomical volumetric images $(\mathrm{T} 1$-weighted $3 \mathrm{D}$ BRAVO, TR/TE $=8.5 / 3.2 \mathrm{~ms}, 1 \times 1 \times 1 \mathrm{~mm}$ voxel size; fluid-attenuated inversion recovery (FLAIR) 3D CUBE, TR/TE $=8500 / 130 \mathrm{~ms}, 1 \times 1 \times 1.4 \mathrm{~mm}$ voxel size), also a 3D pulsed enhanced multiplane tagged continuous ASL (eASL) image set was acquired $\left(\mathrm{TR} / \mathrm{TE}=5917 \mathrm{~ms} / 12.4 \mathrm{~ms}\right.$, bandwidth $976.6 \mathrm{~Hz} /$ pixel, flip angle $111^{\circ}$, time acquisition = 9:09 min $)$ with 26 contiguous slices of $5.5 \mathrm{~mm}$ slice thickness, with voxel size $1.72 \times 1.72 \times 5.5 \mathrm{~mm}$.

ASL images were corrected for arterial transit time (ATT), resulting in transit corrected flow (TCF) images, using vendor-specific software. Because of the noise content, an additional isotropic Gaussian smoothing of $6 \mathrm{~mm}$ was applied on the ATT ASL images before analysis.

\section{Visual qualitative analysis of ${ }^{18}$ F-FDG PET and ASL images}

A qualitative visual analysis was performed on both the ${ }^{18} \mathrm{~F}-\mathrm{FDG}-\mathrm{PET}$ and ASL processed images. Prior to analysis, reconstructed images were fully anonymized and randomly number-coded per data type (differently for ASL and ${ }^{18}$ F-FDG) and processed using the CortexID Suite (GE Healthcare, Chicago, IL, USA). This software allows automatic spatial normalization after which data can be represented in 
an orthogonal and surface rendered way. Two experienced nuclear medicine physicians (K.V.L. and or the working diagnosis at time of ${ }^{18} \mathrm{~F}$-FDG PET. Fig. 1 shows an example of orthogonal slices and surface rendered ${ }^{18}$ F-FDG PET and ASL TCF images for a typical healthy control and AD patient. Both readers visually analyzed the images with the following instructions: firstly, to classify the scan as either 'normal' or 'abnormal'; secondly, if rated abnormal, to classify according to a differential diagnosis from the observed metabolic or flow pattern, in either AD, FTD, LBD or 'other'. The observers were also asked to score activity or flow abnormalities in relevant regional areas using a 4point scale: normal (4), mildly decreased (3), moderately decreased (2) or severely decreased (1). This rating was applied to the following brain regions (left and right): frontal, temporal, parietal and occipital cortex, precuneus, striatum, thalamus and cerebellum. Finally, observers gave a confidence rating for their normal/abnormal and differential diagnosis classification: very uncertain (1), rather uncertain (2), rather certain (3), certain (4).

The observer classifications were analyzed by direct comparison to the ground truth, hereby calculating the diagnostic accuracy (i.e. sensitivity and specificity) for both modalities and for both observers. Within each modality, measures of sensitivity and specificity calculated for each individual observer were subsequently averaged to compare the abnormality intensity score obtained with both modalities. The interobserver agreement was reported with the Fleiss kappa coefficient a, where a $\square$ value between 0.21 and 0.40 represents fair agreement, and 0.61 and 0.80 represents substantial agreement. Abnormality intensity score and confidence scores were plotted on the 4-point scale for both ASL and ${ }^{18} \mathrm{~F}-\mathrm{FDG}$ for all observed regions/scans, and standardized errors were calculated to determine significant rated intensity differences between both techniques.

\section{Semiquantitative VOI analysis}

A volume of interest VOI-based analysis was performed using PMOD (version 3.8, PMOD Inc. Zürich, Switzerland). 3D T1 MRI, ${ }^{18}$ F-FDG PET and eASL images were rigidly matched to account for potential within-scan movement. After T1-based spatial normalization using the PNEURO tool in PMOD (default 
parameters for warping), 83 predefined VOIs from the Hammers atlas [24] were used. Then, larger posterior cingulate, striatum, thalamus and cerebellum). Z-score VOI data were derived by comparing patient VOI data to the mean and SD from the control data $(\mathrm{Z}$-score $=(\mathrm{VOI}($ patient $)-\mathrm{VOI}$ mean(controls))/VOI SD(controls)). Patient scans were classified as normal/abnormal when any composite VOI Z-score was more than 1.5 SD lower than controls. A comparison between eASL and ${ }^{18} \mathrm{~F}$-FDG Z-score data for the patients was performed similar as with the visual grading, i.e. by plotting these for all patients (according to final diagnosis) to determine if data were correlated and investigate the magnitudes of Z-scores for eASL and ${ }^{18} \mathrm{~F}-\mathrm{FDG}$ as indicator of sensitivity to detect hypoperfusion or hypometabolism relative to the variability in controls.

\section{Voxel-based analysis}

A voxel-based group analysis was conducted using Statistical Parametric Mapping (SPM12, Welcome Trust Center for Neuroimaging, London, UK), implemented in MatLab (R2017b, The MathWorks Inc, Natick, MA, USA) to determine the group differences in glucose metabolism and blood flow, and investigate the differences in SPM statistical sensitivity for both techniques.

For this subanalysis, the coregistered image sets were spatially normalized to Montreal Neurological Institute (MNI) space using the SPM TPM template and non-rigid registration with default parameters (16 iterations). Isotropic Gaussian smoothing with full width at half-maximum (FWHM) of $8 \mathrm{~mm}$ was performed in a voxel matrix of $2 \times 2 \times 2 \mathrm{~mm}$. Images were analyzed using proportional scaling to the average grey matter activity. Group analysis was performed at cluster level of $\mathrm{p}<0.05$ (FWE-corrected), with a peak height threshold of $p_{\text {height }}$ of 0.01 (or more stringent), extent threshold ( $k_{\text {ext }}$ ) of 20 voxels. Data patterns were compared for both techniques in the AD and FTD diagnostic subgroups to allow assessment of the average pattern and cluster intensity.

Conventional statistical analysis was performed in SPSS (IBM SPSS Statistics for Macintosh, Version 25.0, Armonk, NY, USA). 


\section{RESULTS}

\section{Patient characteristics}

Patients and controls were age- and gender matched $\left(p=0.48, \mathrm{Chi}^{2} p=0.9\right.$, respectively). The mean MMSE score in the patient group was 24.1 ( \pm 5.5, range: 11-30; available in 23/27 patients), for the CON group this was $29.3 \pm 0.8$ (range: $28-30$ ). The working diagnosis for the patient group is given in Table 1 and consisted of: 8 AD, 2 FTD, 1 LBD, 1 cerebellar variant of multiple system atrophy (MSAc), 1 motor neuron disorder (MND), 1 traumatic brain injury (TBI) and 13 patients with no clear prePET arguments for a neurodegenerative cause for the appeared cognitive complaints (NND = no arguments for neurodegenerative disorder). The final diagnosis after a period of follow-up (follow-up range: 3 - $18 \mathrm{mo}$ ) was different from the working diagnosis at the clinical ${ }^{18} \mathrm{~F}-\mathrm{FDG}$ PET request in 6 subjects (Table 1): NND > AD, MND > NND, AD > NND (post-cerebrovascular accident dementia), $\mathrm{AD}>$ parkinson-related dementia, FTD > FTD+MND.

\section{Visual analysis of ${ }^{18}$ F-FDG PET and ASL images}

Diagnostic accuracy of the visual read of ${ }^{18} \mathrm{~F}$-FDG PET and ASL for the two readers is given in Table 2. For differentiating a pathological (neurodegenerative) from a normal (no arguments for neurodegenerative pattern, NND) scan, the mean sensitivity of ${ }^{18}$ F-FDG PET was 0.93 and equal for both readers, while a substantial difference between readers was noted in specificity $(0.86$ vs 0.53 , mean 0.70). For ASL, the mean sensitivity dropped significantly to $0.64(p=0.03)$ and remained relatively consistent between readers ( 0.71 vs 0.57$)$; also specificity was consistent $(0.74$ vs 0.67 ; mean 0.71$)$.

Furthermore, for the second and much harder task to assign a specific differential diagnosis, of the 14 pathological ${ }^{18}$ F-FDG PET images, 9 were diagnosed correctly by the first reader $(0.64)$, and $8(0.57)$ by the second reader (average correct classification 0.61). For the ASL images, $7(0.50)$ and $4(0.29)$ out of 14 images were classified correctly by the first and second reader, respectively (average correct classification $0.39 ; p>0.05)$. The corresponding Cohen's Kappa coefficients for interobserver agreement between modalities were 0.34 (SE $0.15,95 \%$ CI 0.04 to 0.65 ) and 0.20 (SE $0.15,95 \%$ CI 0.11 to 0.50 ) for ${ }^{18} \mathrm{~F}-\mathrm{FDG}$ PET and ASL, respectively, suggesting that the agreement between the observers was better for ${ }^{18} \mathrm{~F}-\mathrm{FDG}$ PET than for ASL. 
Abnormality intensity rating: When the readers were asked to observe and score the ${ }^{18} \mathrm{~F}$-FDG

activity/flow abnormality in the relevant brain regions, the average visual intensity rating was comparable between ${ }^{18} \mathrm{~F}$-FDG PET and ASL in the frontal, parietal, temporal cortex, precuneus and cerebellum for each patient group $(p>0.05)$ (Fig. 2, Supplementary Fig. 1). Contrarily, average abnormality scores were significantly lower in the occipital lobe, thalamus and striatum for ASL versus ${ }^{18}$ F-FDG PET data (“moderately-mildly decreased" with ASL vs "normal” with ${ }^{18}$ F-FDG PET) (Fig. 2, Supplementary Fig. 1). These findings were also confirmed by the standardized residuals. For the patient groups, the standardized residuals were more than 2 , indicating ${ }^{18}$ F-FDG-based intensity rating was significantly higher than ASL-based intensity rating in 5 patients, and vice versa in one other patients.

Confidence rating: Considering the regional confidence rating for the left and right observed brain regions, the degree of diagnostic confidence for ${ }^{18} \mathrm{~F}$-FDG PET and ASL was comparable in the frontal cortex, parietal cortex, precuneus and cerebellum (Supplementary Fig. 2.a). However, the readers were less confident in scoring ASL-based blood flow compared to glucose metabolism in the temporal cortex, occipital cortex, striatum and thalamus in any group of patients with suspected diagnosis of dementia (NND, AD, FTD, LBD and OTHER), as well as for the control group (Supplementary Fig. 2.b).

\section{Regional pattern scoring: semiquantitative VOI analysis}

In a second step, the subjects were classified as normal/abnormal scans based on the composite VOI Zscore assessments. The sensitivity and specificity of ${ }^{18} \mathrm{~F}-\mathrm{FDG}$ PET-based distinction was 0.79 and 0.63 , respectively. ASL yielded a lower rate of correct differential diagnosis in normal vs. abnormal cases, obtaining a sensitivity of $0.57(p<0.001)$. On the other hand, the rate of true negatives was significantly higher with ASL (specificity $=0.81 ; p<0.001$ ). In Fig. 3 , the Z-score data are plotted for both ASL and ${ }^{18} \mathrm{~F}-\mathrm{FDG}$ for all observed regions/scans. Overall, the standardized residuals revealed no significant Zscore-related differences between both techniques (frontal cortex: $-0.53 \pm 0.83$; temporal cortex: -0.53 \pm 0.45 ; parietal cortex: $-0.50 \pm 1.18$; occipital cortex: $0.64 \pm 0.82$; posterior cingulate: $-0.61 \pm 1.03$; striatum: $-0.69 \pm 0.85$; thalamus : $-0.66 \pm 0.86$; cerebellum: $0.70 \pm 0.99) .{ }^{18} \mathrm{~F}-\mathrm{FDG}$-based VOI Z-scores were significantly higher than ASL-based VOI Z-scores in 5\% of the regional values. 


\section{Voxel-based analysis}

Voxel-wise statistical analysis results of AD and FTD patients compared to CON are shown in Fig. 4.

Group differences in glucose metabolism and blood flow between the AD diagnostic subgroup compared to CON, showed that ${ }^{18}$ F-FDG PET and ASL result in similar quantitative hypometabolism/hypoperfusion localized mainly in the mid-cingulate, posterior cingulate-precuneus cortices and parietotemporal areas, typical of $\mathrm{AD}$ (Fig. 4.a). Although an agreement between the hypoperfusion and hypometabolism maps was confirmed by a $2^{\text {nd }}$ level factorial analysis (positive effect of condition, with a peak height threshold of $p_{\text {height }}$ of 0.001 and $k_{\text {ext }}$ of 20 voxels), SPM statistical cluster intensity and extent was greater with ${ }^{18} \mathrm{~F}-\mathrm{FDG}$ PET $\left(p_{\mathrm{FWE}}<0.05\right.$ at cluster level), compared to ASL data. When considering the FTD diagnostic subgroup vs CON, the extent of the anteromedial temporal and inferior frontal cortical abnormality was larger on ${ }^{18}$ F-FDG PET than on ASL (Fig. 4.b). A summary of the localization of the clusters, $p$ and $t$ scores for the AD vs CON and FTD vs CON group comparison is shown in Supplementary Table 1 and Supplementary Table 2, respectively. 


\section{DISCUSSION}

In the past few years, several single-centre studies have reported good correlations between observed hypoperfusion in ASL and hypometabolism in ${ }^{18} \mathrm{~F}$-FDG PET in patients with AD or FTD, suggesting ASL as a potential alternative to ${ }^{18} \mathrm{~F}-\mathrm{FDG}$ PET in the diagnosis of neurodegenerative dementia [17-23]. However, the diagnostic value of ASL in clinical conditions has yet to be accurately confirmed, based on the inconclusive evidence from these group comparisons. Indeed, the reported sensitivity and specificity of ASL compared to ${ }^{18} \mathrm{~F}-\mathrm{FDG}$ PET studies largely varies among the studies, which is likely due to differences in ASL techniques, type of comparative analysis, varying PET and MR conditions or heterogeneity of small cohorts of patients.

In this direct head-to-head comparison between multiplane tagged, pulsed enhanced multiplane tagging ASL (eASL) and ${ }^{18} \mathrm{~F}-\mathrm{FDG}$ PET, set in a true clinical context of referrals for cognitive decline in a tertiary setting, we aimed at a comprehensive evaluation of the diagnostic performance of the two techniques by using a simultaneous PET/MR system and a combination of visual and semiquantitative analysis, as is done in standard clinical practice. The main finding of this study was that eASL could be considered as a potential alternative to ${ }^{18} \mathrm{~F}$-FDG PET to assess neurodegeneration in patients with cognitive impairment when the latter is unavailable, or in case dual-parameter evaluation (e.g. amyloid or tau PET + eASL-MR) can be done to provide simultaneous $\beta$ amyloid deposition, pathologic tau, and neurodegeneration (ATN) classification [25]. Within the setting of this study, ${ }^{18} \mathrm{~F}-\mathrm{FDG}$ PET should still be seen as the primary choice, as it performed better compared to eASL in terms of sensitivity, reader confidence, effect size and lower variability in key regions in dementia diagnosis. These findings are in line with previous work on perfusion SPECT and ${ }^{18}$ F-FDG PET in the individual diagnosis of Alzheimer's dementia, where 15-20\% higher sensitivity and accuracy were found in favour of ${ }^{18} \mathrm{~F}-\mathrm{FDG}$ PET [26]. This had physiological (earlier glucose metabolic decline) as well as technical reasons (lower SPECT spatial resolution).

In order to compare the diagnostic accuracy of ${ }^{18} \mathrm{~F}-\mathrm{FDG}$ PET and eASL in detecting functional abnormalities associated with dementia, we first performed a standardized qualitative visual analysis. This resulted in equal specificity for differentiating normal and abnormal scans for the two modalities, but in a higher sensitivity for ${ }^{18} \mathrm{~F}$-FDG PET compared to eASL. Considering the previous comparative 
qualitative analyses using visual rating methods in AD and/or FTD patients, previous work found similar findings of lower observer agreement for ASL but matched sensitivity between the two modalities $[19,21,23]$. Fällmar et al. noted a higher specificity and positive predictive value using ASL, but a higher sensitivity and accuracy using ${ }^{18}$ F-FDG PET images, when visually assessing ASL-based and ${ }^{18}$ F-FDGbased Z-maps in controls and patients with AD and FTD [17]. Since our study measured diagnostic performance of ASL and ${ }^{18} \mathrm{~F}$-FDG PET in various types of potential neurodegenerative disorders, rather than exclusively AD [23] and/or FTD [19,21], it is challenging to directly compare our results with these prior investigations.

Furthermore, for the second and much harder task to assign a specific differential diagnosis, including blinded evaluation of screened healthy controls, we reported a higher percentage of correct classification with ${ }^{18} \mathrm{~F}$-FDG PET than corresponding ASL image data. We observed a comparable intensity reduction of cerebral perfusion and metabolism, predominantly in the parietal and posterior cingulate cortex in $\mathrm{AD}$. In other regions that are normometabolic/normoperfused in $\mathrm{AD}$, such as the primary visual cortex, cerebellum and subcortical regions, abnormality and confidence scores were lower with ASL which may be due to lower values in normal individuals (see for example Fig. 1.A), watershed artefacts or higher variability across the subcortical regions $[27,28]$. It is known that central arterial transit times (ATT) are shorter than for the cortex, and that, even for multiplane tagging approaches such as applied in our work, this difference may give rise to an underestimation of $\mathrm{CBF}$ as was shown in direct ${ }^{15} \mathrm{O}$ $\mathrm{H}_{2} \mathrm{O}-\mathrm{PET}$ versus eASL head to head comparative studies (Ishii et al, 2019; unpublished results). This regional ASL and FDG differences can also be observed in our current work. Fig. 5 shows the hypoperfusion in subcortical regions (such as basal ganglia and thalamus) in both healthy controls and AD patients, obtained comparing ASL vs ${ }^{18} \mathrm{~F}-\mathrm{FDG}$ PET. In pathological conditions, such as AD, also changes in ATT can give rise to alterations in the flow maps $[29,30]$.

The semiquantitative VOI- and voxel-based analyses at the group-level provided supportive findings that were in line with the visual analysis. The regional Z-score approach confirmed the higher sensitivity of ${ }^{18}$ F-FDG PET compared to ASL in detecting abnormalities. On the other hand, the specificity was found to be higher with ASL compared to ${ }^{18} \mathrm{~F}$-FDG PET, resulting in a similar overall accuracy between 
the two modalities for the classification into normal versus neurodegenerative abnormalities, which is

In this context, it is of importance to note that the two reviewers were nuclear medicine specialists not trained for neuroradiology, and thus had more experience with clinical assessment of ${ }^{18} \mathrm{~F}-\mathrm{FDG}$ PET. We do not consider this a disadvantage or study design problem, as both had also long-term experience in evaluating clinical routine perfusion SPECT images, and few neuroradiologists perform visual assessment of ASL-MRI in a routine setting of neurodegeneration/dementia workup. Nevertheless, the diagnostic performance of ASL in the visual analysis could be higher if an elaborated reader training was implemented, or the visual analysis was complemented by availability of statistical Z-score maps on a rendered surface projection, similarly to the work of Fällmar et al. [17]. In the latter study, the sensitivity of ${ }^{18}$ F-FDG-based Z-maps was still higher than in the corresponding ASL-based images [17], and the visual findings in our study were also corroborated by semiquantitative analyses with similar results.

When evaluating voxel-based group differences in glucose metabolism and blood flow in AD compared to controls, we found a similar spatial hypometabolism/hypoperfusion pattern localized in the posterior cingulate, precuneus and parietotemporal areas [31,32], although both cluster intensity and extent of the AD pattern was greater with ${ }^{18} \mathrm{~F}$-FDG PET compared to ASL, again indicating more robustly detectable abnormalities in this subgroup. Similarly, anteromedial temporal and prefrontal abnormalities $[17,19,31]$ were more pronounced in FTD for ${ }^{18} \mathrm{~F}-\mathrm{FDG}$ PET versus ASL. In a recent simultaneous PET/MR study comparing ASL and ${ }^{18}$ F-FDG in AD and mild cognitive impairment (MCI) [33], a voxelwise analysis also revealed similar regional and quantitative abnormalities between ${ }^{18} \mathrm{~F}-\mathrm{FDG}$ PET and ASL, and ASL images provided a reduced extent compared to ${ }^{18} \mathrm{~F}-\mathrm{FDG}$ PET, in line with our findings. In patients with MCI, a voxel-wise analysis revealed no CBF reductions between MCI and controls in the study of Riederer et al. [33], in contrast to ${ }^{18} \mathrm{~F}$-FDG PET with quantitative hypometabolism in the precuneus, a brain region known to be one of the first affected in MCI due to AD [34]. A significantly lower sensitivity of ASL Z-maps compared to ${ }^{18} \mathrm{~F}$-FDG PET Z-maps in discrimination of AD+FTD as reported by Fällmar et al. [17], also confirms that regional CBF impairment is milder and/or occurs at a later disease stage compared to regional hypometabolism. 
The additional value of the current study over published data can be summarized by the following strengths. First of all, a head-to-head simultaneous and prospective comparison between ${ }^{18} \mathrm{~F}-\mathrm{FDG}$ PET and ASL was performed in a true clinical context of patients with cognitive impairment referred for exclusion/confirmation of a neurodegenerative disorder after careful clinical and paraclinical workup. In contrast to previous comparative studies where the patient cohort was selected retrospectively, timing discrepancies between both scans (disease progression) and selection bias (further imaging when inconclusive previous imaging investigations) may have played a role. The majority of the studies comparing PET and ASL have been performed on both separate PET/CT and MR systems and separate occasions, with an interval between both exams ranging from a few days [21] up to 6 months [17,22,31,35-37]. Simultaneous comparative PET/MR studies in dementia are rare [19,33,38]. Also, the vast majority of these studies are principally based on voxel-wise image analysis at a group level, which is not easily translatable to actual impact in clinical routine. Moreover, we included an age and gender matched healthy control set acquired on the same instrumentation that was evaluated in a blinded fashion and the heterogeneity of final diagnoses represents a true clinical scala of uncertain cases with cognitive impairment.

A major limitation of this study is the relatively small patient population that underwent additional PET/MR scanning aside from the standard-of-care PET/CT scan. Nevertheless, the conclusions that can be drawn from this study, are already significant and clearly show the differences between both techniques with the current utilized instrumentation. Secondly, ground truth diagnosis was based on clinical assessment including all routinely available information and disease follow-up. It is known that in the setting of a tertiary memory clinic, diagnostic accuracy can approach 90\% [26], but in large proportions the final diagnosis may change with advance biomarkers such as amyloid PET [39]. Evaluations with CSF A $\beta$ and tau measurement and/or amyloid PET were only available for a small subgroup of patients at final follow-up. Another limitation is the eASL imaging acquisition used. It is known that the interindividual variance in ASL perfusion is large compared to the variance of standardised uptake ratios in ${ }^{18}$ F-FDG PET [40] and the sensitivity of eASL could have been improved using higher channel head coils with more (32/64) receiver channels, that may offer up to twice the signal-to-noise ratio (SNR) compared to an 8 channel coils. Improvement of SNR in eASL images may 
likely decrease the variability across brain regions. It remains to be proven that incorporation of the the majority of the previous studies that have compared the diagnostic performance of ASL versus ${ }^{18} \mathrm{~F}$ FDG PET in dementia used a similar standard receiver coil [17,19,21,22].

As the data were acquired before implementation and validation of the Zero Echo Time (ZTE) technique, which is now standardly used for individual MR-based attenuation map generation on the GE Signa PET/MR, we used the vendor-supplied MRAC correction, which is known to give rise to a small but significant craniocaudal gradient in the images with an underestimation of the infratentorial ${ }^{18}$ F-FDG activity [41]. It is unlikely that this would have driven the observed visual classification and semiquantitative evaluation however, as it was also applied to the control data set. Finally, we did not correct for partial volume effects since the primary aim of the study was to resemble clinical routine evaluation as much as possible. Such correction was also not performed on the perfusion maps nor in most previous studies in comparing ${ }^{18} \mathrm{~F}-\mathrm{FDG}$ and ASL $[23,33,42]$.

In conclusion, in this current direct prospective comparison between ${ }^{18} \mathrm{~F}-\mathrm{FDG}$ PET and eASL in a true clinical context of differentiating neurodegenerative versus non-neurodegenerative classification of cognitively impaired patients, as well as differentiation in dementia subtypes, we found that, on the GE Signa PET/MR with multiplane tagging enhanced eASL, ${ }^{18}$ F-FDG PET outperforms eASL in terms of higher sensitivity, reader confidence, effect size and lower variability in key regions in dementia diagnosis. When performing a semiquantitative analysis, a similar diagnostic accuracy between the two modalities was obtained. As such, eASL with appropriate semiquantitative evaluation and comparison to normal data, may complement ${ }^{18} \mathrm{~F}-\mathrm{FDG}$ PET or be an adjunct parameter to assess the $\mathrm{N}$ (neuronal injury) status in patients suspected for dementia where in case of simultaneous acquisition, PET can be directed towards amyloid or tau assessment. 


\section{Compliance with Ethical Standards}

Funding: Jenny Ceccarini and Donatienne Van Weehaeghe are postdoctoral and $\mathrm{PhD}$ fellows of the Research Foundation Flanders (FWO) respectively. Rik Vandenberghe and Koen Van Laere are Senior Clinical Investigators of the FWO.

Conflict of Interest: The authors declare that they have no conflict of interest.

Ethical approval: All procedures performed in studies involving human participants were in accordance with the ethical standards of the institutional and/or national research committee and with the 1964 Helsinki declaration and its later amendments or comparable ethical standards.

Informed consent: Informed consent was obtained from all individual participants included in the study. 


\section{REFERENCES}

1. Drew L. An age-old story of dementia. Nature. 2018;559:S2-3.

2. Prince M, Bryce R, Albanese E, Wimo A, Ribeiro W, Ferri CP. The global prevalence of dementia: A systematic review and metaanalysis. Alzheimer's Dement.2013;9:63-75.e2.

3. Shivamurthy VK, Tahari AK, Marcus C, Subramaniam RM, Vkn S. Brain FDG PET and the Diagnosis of Dementia. AJR Am J Roentgenol. 2015;204(1):W76-85.

4. Herman AO and Molano JR. For Patients with Cognitive Impairment, Amyloid PET Leads to Changes in Clinical Management. NEJM J Watch. 2019 Apr 19.

5. Villemagne VL, Doré V, Bourgeat P, Burnham SC, Laws S, Salvado O, et al. A $\beta$-amyloid and Tau Imaging in Dementia. Semin Nucl Med . W.B. Saunders; 2017;47:75-88.

6. Okamura N, Harada R, Furumoto S, Tago T, Yanai K, Arai H, et al. Advances in the development of tau PET radiotracers and their clinical applications. Ageing Res Rev. 2016;30:107-13.

7. Mallik A, Drzezga A, Minoshima S. Clinical Amyloid Imaging. Semin Nucl Med . W.B. Saunders; 2017;47:31-43.

8. Morbelli S, Garibotto V, Van De Giessen E, Arbizu J, Chételat G, Drezgza A, et al. A Cochrane review on brain $\left[{ }^{18} \mathrm{~F}\right] \mathrm{FDG}$ PET in dementia: limitations and future perspectives. Eur J Nucl Med Mol Imaging. 2015;42:1487-91.

9. Garibotto V, Herholz K, Boccardi M, Picco A, Varrone A, Nordberg A, et al. Clinical validity of brain fluorodeoxyglucose positron emission tomography as a biomarker for Alzheimer's disease in the context of a structured 5-phase development framework. Neurobiol Aging. 2017;52:183-95.

10. Foster NL, Heidebrink JL, Clark CM, Jagust WJ, Arnold SE, Barbas NR, et al. FDG-PET improves accuracy in distinguishing frontotemporal dementia and Alzheimer's disease. Brain. 2007;130:261635 .

11. Tosun D, Schuff N, Rabinovici GD, Ayakta N, Miller BL, Jagust W, et al. Diagnostic utility of ASLMRI and FDG-PET in the behavioral variant of FTD and AD. Ann Clin Transl Neurol. 2016;3:740-51. 12. Jueptner M, Weiller C. Review: does measurement of regional cerebral blood flow reflect synaptic activity? Implications for PET and fMRI. Neuroimage. 1995;2:148-56.

13. Du AT, Jahng GH, Hayasaka S, Kramer JH, Rosen HJ, Gorno-Tempini ML, et al. Hypoperfusion in frontotemporal dementia and Alzheimer disease by arterial spin labeling MRI. Neurology. 2006;67:1215-20.

14. Raji CA, Lee C, Lopez OL, Tsay J, Boardman JF, Schwartz ED, et al. Initial experience in using continuous arterial spin-labeled MR imaging for early detection of Alzheimer disease. AJNR Am J Neuroradiol. 2010;31:847-55.

15. Kaneta T, Katsuse O, Hirano T, Ogawa M, Shihikura-Hino A, Yoshida K, et al. Voxel-wise correlations between cognition and cerebral blood flow using arterial spin-labeled perfusion MRI in patients with Alzheimer's disease: a cross-sectional study. BMC Neurol. 2017;17:91. 
16. Liu P, Uh J, Devous MD, Adinoff B, Lu H. Comparison of relative cerebral blood flow maps using pseudo-continuous arterial spin labeling and single photon emission computed tomography. NMR Biomed. 2012;25:779-86.

17. Fällmar D, Haller S, Lilja J, Danfors T, Kilander L, Tolboom N, et al. Arterial spin labeling-based Z-maps have high specificity and positive predictive value for neurodegenerative dementia compared to FDG-PET. Eur Radiol. 2017;27:4237-46.

18. Verfaillie SCJ, Adriaanse SM, Binnewijzend MAA, Benedictus MR, Ossenkoppele R, Wattjes MP, et al. Cerebral perfusion and glucose metabolism in Alzheimer's disease and frontotemporal dementia: two sides of the same coin? Eur Radiol. 2015;25:3050-9.

19. Anazodo UC, Finger E, Kwan BYM, Pavlosky W, Warrington JC, Günther M, et al. Using simultaneous PET/MRI to compare the accuracy of diagnosing frontotemporal dementia by arterial spin labelling MRI and FDG-PET. NeuroImage Clin. 2018;17:405-14.

20. Riederer I, Bohn KP, Preibisch C, Wiedemann E, Zimmer C, Alexopoulos P, et al. Alzheimer Disease and Mild Cognitive Impairment: Integrated Pulsed Arterial Spin-Labeling MRI and 18 F-FDG PET. Radiology. 2018;288:198-206.

21. Weyts K, Vernooij M, Steketee R, Valkema R, Smits M. Qualitative agreement and diagnostic performance of arterial spin labelling MRI and FDG PET-CT in suspected early-stage dementia: Comparison of arterial spin labelling MRI and FDG PET-CT in suspected dementia. Clin Imaging. $2017 ; 45: 1-7$.

22. Tosun D, Schuff N, Rabinovici GD, Ayakta N, Miller BL, Jagust W, et al. Diagnostic utility of ASLMRI and FDG-PET in the behavioral variant of FTD and AD. Ann Clin Transl Neurol. 2016;3:740-51. 23. Musiek ES, Chen Y, Korczykowski M, Saboury B, Martinez PM, Reddin JS, et al. Direct Comparison of FDG-PET and ASL-MRI in Alzheimer's Disease. Alzheimers Dement. 2012;8:51-9. 24. Hammers A, Allom R, Koepp MJ, Free SL, Myers R, Lemieux L, et al. Three-dimensional maximum probability atlas of the human brain, with particular reference to the temporal lobe. Hum Brain Mapp. 2003;19:224-47.

25. Jack CR, Bennett DA, Blennow K, Carrillo MC, Dunn B, Haeberlein SB, et al. NIA-AA Research Framework: Toward a biological definition of Alzheimer's disease. Alzheimers Dement. 2018;14:53562.

26. Bohnen NI, Djang DSW, Herholz K, Anzai Y, Minoshima S. Effectiveness and safety of 18F-FDG PET in the evaluation of dementia: a review of the recent literature. J Nucl Med. 2012;53:59-71.

27. Deibler AR, Pollock JM, Kraft RA, Tan H, Burdette JH, Maldjian JA. Arterial spin-labeling in routine clinical practice, part 1: technique and artifacts. AJNR Am J Neuroradiol. 2008;29:1228-34.

28. Grade M, Hernandez Tamames JA, Pizzini FB, Achten E, Golay X, Smits M. A neuroradiologist's guide to arterial spin labeling MRI in clinical practice. Neuroradiology. 2015;57:1181-202.

29. Yoshiura T, Hiwatashi A, Yamashita K, Ohyagi Y, Monji A, Takayama Y, et al. Simultaneous Measurement of Arterial Transit Time, Arterial Blood Volume, and Cerebral Blood Flow Using Arterial 
Spin-Labeling in Patients with Alzheimer Disease. Am J Neuroradiol. 2009; 30:1388-93.

30. Tang X, Cai F, Ding D-X, Zhang L-L, Cai X-Y, Fang Q. Magnetic resonance imaging relaxation time in Alzheimer's disease. Brain Res Bull. 2018;140:176-89.

31. Verfaillie SCJ, Adriaanse SM, Binnewijzend MAA, Benedictus MR, Ossenkoppele R, Wattjes MP, et al. Cerebral perfusion and glucose metabolism in Alzheimer's disease and frontotemporal dementia: two sides of the same coin? Eur Radiol. 2015;25:3050-9.

32. Chen Y, Wolk DA, Reddin JS, Korczykowski M, Martinez PM, Musiek ES, et al. Voxel-level comparison of arterial spin-labeled perfusion MRI and FDG-PET in Alzheimer disease. Neurology. 2011;77:1977-85.

33. Riederer I, Bohn KP, Preibisch C, Wiedemann E, Zimmer C, Alexopoulos P, et al. Alzheimer Disease and Mild Cognitive Impairment: Integrated Pulsed Arterial Spin-Labeling MRI and ${ }^{18}$ F-FDG PET. Radiology. 2018;288:198-206.

34. Drzezga A, Lautenschlager N, Siebner H, Riemenschneider M, Willoch F, Minoshima S, et al. Cerebral metabolic changes accompanying conversion of mild cognitive impairment into Alzheimer's disease: a PET follow-up study. Eur J Nucl Med Mol Imaging. 2003;30:1104-13.

35. Fällmar D, Lilja J, Velickaite V, Danfors T, Lubberink M, Ahlgren A, et al. Visual Assessment of Brain Perfusion MRI Scans in Dementia: A Pilot Study. J Neuroimaging. 2016;26:324-30.

36. Verclytte S, Lopes R, Lenfant P, Rollin A, Semah F, Leclerc X, et al. Cerebral Hypoperfusion and Hypometabolism Detected by Arterial Spin Labeling MRI and FDG-PET in Early-Onset Alzheimer's Disease. J Neuroimaging. 2016;26:207-12.

37. Teune LK, Renken Phd RJ, De Jong BM, Willemsen AT, Van Osch MJ, Roerdink JBTM, et al. Parkinson's disease-related perfusion and glucose metabolic brain patterns identified with PCASL-MRI and FDG-PET imaging. Neuroimage Clin. 2014;5:240-4.

38. Douglas D, Goubran M, Wilson E, Xu G, Tripathi P, Holley D, et al. Correlation between arterial spin labeling MRI and dynamic FDG on PET-MR in Alzheimer's disease and non-Alzhiemer's disease patients. EJNMMI Phys. 2015;2:A83.

39. Rabinovici GD, Gatsonis C, Apgar C, Chaudhary K, Gareen I, Hanna L, et al. Association of Amyloid Positron Emission Tomography With Subsequent Change in Clinical Management Among Medicare Beneficiaries With Mild Cognitive Impairment or Dementia. JAMA.2019;321:1286.

40. Henriksen OM, Larsson HBW, Hansen AE, Grüner JM, Law I, Rostrup E. Estimation of intersubject variability of cerebral blood flow measurements using MRI and positron emission tomography. J Magn Reson Imaging. 2012;35:1290-9.

41. Schramm G, Koole M, Willekens SMA, Rezaei A, Van Weehaeghe D, Delso G, et al. Regional accuracy of ZTE-based attenuation correction in static and dynamic brain PET/MR. 2018; arXiv: 1806.03481

42. Ferreira LK, Diniz BS, Forlenza O V., Busatto GF, Zanetti M V. Neurostructural predictors of Alzheimer's disease: A meta-analysis of VBM studies. Neurobiol Aging. 2011;32:1733-41. 


\title{
FIGURE TITLES and LEGENDS
}

\begin{abstract}
Figure 1. Typical examples of orthogonal and 3D-surface rendered eASL-MR and ${ }^{18}$ F-FDG PET images of a healthy control (female, 40y; panel A) and subject with AD (male, 63y; panel B), respectively. Orthogonal and surface rendered images are scaled to the relative maximum value. Both images were classified concordantly and in the correct diagnostic class.
\end{abstract}

Figure 2. Visual rating of regional intensity for ${ }^{18} \mathrm{~F}-\mathrm{FDG}$ PET and ASL Average rating $(1=$ severely decreased, $2=$ moderately decreased, $3=$ mildly decreased, $4=$ normal $)$ for right and left observed brain areas (frontal, parietal, temporal, occipital cortex, striatum, thalamus, precuneus and cerebellum). The ellipses indicates the average intensity rating corresponding to the occipital cortex, thalamus and striatum with the highest disagreement between both modalities. The intermittent line indicates the identity line.

Abbreviations: CON, healthy controls; NND, no arguments for neurodegenerative disorder; AD, Alzheimer's disease; FTD, frontotemporal dementia; LBD, Lewy body dementia; OTHER, rest of the patient group including motor neuron disease, cerebellar variant of multiple system atrophy, vascular dementia, and traumatic brain injury.

Figure 3. Regional semiquantitative analysis for assessment of neurodegenerative versus nonneurodegenerative scans with ${ }^{18} \mathrm{~F}-\mathrm{FDG}$ PET vs ASL

FDG PET- and ASL-based Z-scores for all observed brain areas (frontal, parietal, temporal, occipital cortex, striatum, thalamus, precuneus and cerebellum)/scans. The intermittent line indicates the identity line.

Abbreviations: NND, no arguments for neurodegenerative disorder; AD, Alzheimer's disease; FTD, frontotemporal dementia; LBD, Lewy body dementia; OTHER, rest of the patient group including motor neuron disease, cerebellar variant of multiple system atrophy, vascular dementia, and traumatic brain injury. 
Figure 4. Results of the SPM group analysis for ${ }^{18}$ F-FDG PET and ASL: a) 30 CON versus 8 AD patients; $b$ ) $30 \mathrm{CON}$ versus 2 FTD patients. Evaluations at $p_{\text {height }}<0.01$ and extend threshold $\mathrm{k}_{\mathrm{ext}}=20$ $\left(2 \times 2 \times 2 \mathrm{~mm}^{3}\right)$ voxels.

Figure 5. Transversal, parasagittal and coronal average eASL-MR (top row) and ${ }^{18}$ F-FDG PET maps (bottom row) for healthy controls (CON) (left panel) and AD patients (right panel). Images are scaled to the global grey matter value.

White triangles indicate eASL $<{ }^{18} \mathrm{~F}-\mathrm{FDG}$ PET in subcortical regions such as in the basal ganglia and thalamus. 
Table 1. Patient demographics and diagnoses.

\begin{tabular}{|c|c|c|c|c|c|c|}
\hline $\begin{array}{l}\text { Patients } \\
\text { number }\end{array}$ & $\begin{array}{c}\text { Age } \\
\text { (years) }\end{array}$ & $\begin{array}{c}\text { Gender } \\
(\mathrm{M} / \mathrm{F})\end{array}$ & MMSE & $\begin{array}{c}\text { Working } \\
\text { diagnosis } \\
\text { at }{ }^{18} \mathrm{~F} \text {-FDG PET }\end{array}$ & Final Dx & $\begin{array}{c}\text { Years of } \\
\text { symptoms prior } \\
\text { to }{ }^{18} \text { F-FDG PET }\end{array}$ \\
\hline 1 & 69 & $\mathrm{~F}$ & 27 & NND & NND & 2.1 \\
\hline 2 & 63 & M & 30 & NND & NND & 2.8 \\
\hline 3 & 55 & $\mathrm{~F}$ & 28 & NND & NND & 2.1 \\
\hline 4 & 76 & M & 26 & $\mathrm{AD}$ & $\mathrm{AD}$ & 6.5 \\
\hline 5 & 40 & $\mathrm{~F}$ & 30 & NND & NND & 1.9 \\
\hline 6 & 47 & $\mathrm{M}$ & 30 & TBI & TBI & 31.4 \\
\hline 7 & 70 & $\mathrm{~F}$ & 23 & FTD & FTD & 0.2 \\
\hline 8 & 68 & $\mathrm{~F}$ & 28 & NND & $\mathrm{AD}^{*}$ & 2.3 \\
\hline 9 & 47 & M & $\mathrm{n} / \mathrm{a}$ & NND & NND & $\mathrm{n} / \mathrm{a}$ \\
\hline 10 & 73 & $\mathrm{~F}$ & 25 & $\mathrm{AD}$ & $\mathrm{AD}$ & 1.0 \\
\hline 11 & 56 & $\mathrm{~F}$ & 11 & MND & NND* & 1.0 \\
\hline 12 & 72 & $\mathrm{M}$ & 18 & $\mathrm{AD}$ & $\begin{array}{c}\text { NND } \\
(\text { post-CVA)* }\end{array}$ & 0.7 \\
\hline 13 & 73 & M & 12 & $\mathrm{AD}$ & $\mathrm{AD}$ & 0.8 \\
\hline 14 & 71 & $\mathrm{~F}$ & 17 & $\mathrm{AD}$ & $\mathrm{AD}$ & 0.7 \\
\hline 15 & 66 & M & 22 & $\mathrm{AD}$ & $\mathrm{AD}$ & 3.2 \\
\hline 16 & 40 & M & 25 & NND & NND & 1.6 \\
\hline 17 & 77 & M & $\mathrm{n} / \mathrm{a}$ & $\mathrm{AD}$ & Parkinsonism* & $\mathrm{n} / \mathrm{a}$ \\
\hline 18 & 77 & $\mathrm{~F}$ & 21 & NND & $\mathrm{AD}^{*}$ & 0.5 \\
\hline 19 & 70 & $\mathrm{~F}$ & 27 & NND & NND & 2.5 \\
\hline 20 & 63 & M & 24 & $\mathrm{AD}$ & $\mathrm{AD}$ & 0.7 \\
\hline 21 & 73 & $\mathrm{M}$ & 29 & MSA-c & MSA-c & 1.9 \\
\hline 22 & 69 & $\mathrm{~F}$ & $\mathrm{n} / \mathrm{a}$ & LBD & LBD & $\mathrm{n} / \mathrm{a}$ \\
\hline 23 & 57 & M & $\mathrm{n} / \mathrm{a}$ & NND & NND & 1.6 \\
\hline 24 & 55 & $\mathrm{~F}$ & 27 & NND & NND & 1.6 \\
\hline 25 & 78 & $\mathrm{~F}$ & 23 & NND & NND & 2.0 \\
\hline 26 & 59 & M & 30 & NND & NND & 1.6 \\
\hline 27 & 71 & $\mathrm{M}$ & 21 & FTD & FTD $(+\mathrm{MND}) *$ & 0.5 \\
\hline
\end{tabular}

Dx, diagnosis; AD, Alzheimer's disease; FTD, frontotemporal dementia; LBD, Lewy body dementia; MND, motor neuron disease; MSA-c, cerebellar variant of multiple system atrophy; CVA, cerebrovascular accident dementia; NND, no arguments for neurodegenerative disorder; TBI, traumatic brain injury; n/a, data not available. * indicates alterations from the working diagnosis (Dx) at the time of ${ }^{18}$ F-FDG PET. 
Table 2. Visual read results for ${ }^{18}$ F-FDG PET and ASL, classified into normal and abnormal neurodegenerative (ND) pattern.

\begin{tabular}{|c|c|c|c|c|}
\hline \multicolumn{5}{|l|}{${ }^{18}$ F-FDG } \\
\hline \multirow[b]{2}{*}{ Final diagnosis } & \multicolumn{2}{|c|}{ PET ND pattern } & \multicolumn{2}{|c|}{ PET normal } \\
\hline & Reader 1 & Reader 2 & Reader 1 & Reader 2 \\
\hline Neurodegenerative disorder (14) & 13 & 13 & 1 & 1 \\
\hline $\begin{array}{l}\text { Normal / no neurodegenerative } \\
\text { disorder }(30 \mathrm{CON}+13)\end{array}$ & 6 & 20 & 37 & 23 \\
\hline Sensitivity & 0.93 & 0.93 & & \\
\hline Specificity & 0.86 & 0.53 & & \\
\hline \multicolumn{5}{|l|}{ ASL } \\
\hline & \multicolumn{2}{|c|}{ ASL ND pattern } & \multicolumn{2}{|c|}{ ASL normal } \\
\hline Final diagnosis & Reader 1 & Reader 2 & Reader 1 & Reader 2 \\
\hline Neurodegenerative disorder & 10 & 8 & 4 & 6 \\
\hline $\begin{array}{l}\text { Normal / no neurodegenerative } \\
\text { disorder }\end{array}$ & 11 & 14 & 32 & 29 \\
\hline Sensitivity & 0.71 & 0.57 & & \\
\hline Specificity & 0.72 & 0.67 & & \\
\hline
\end{tabular}




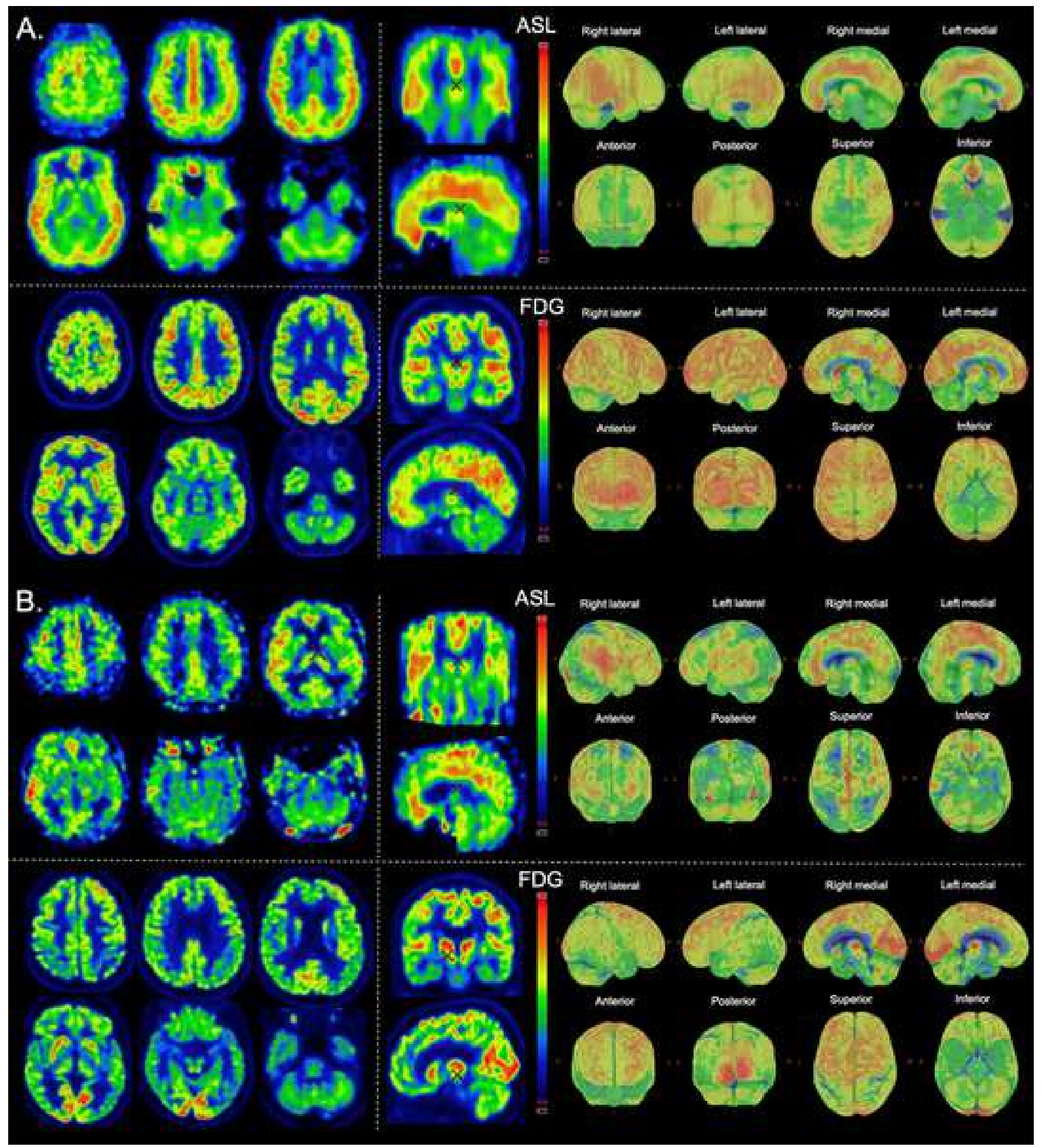




\section{Regional intensity rating}

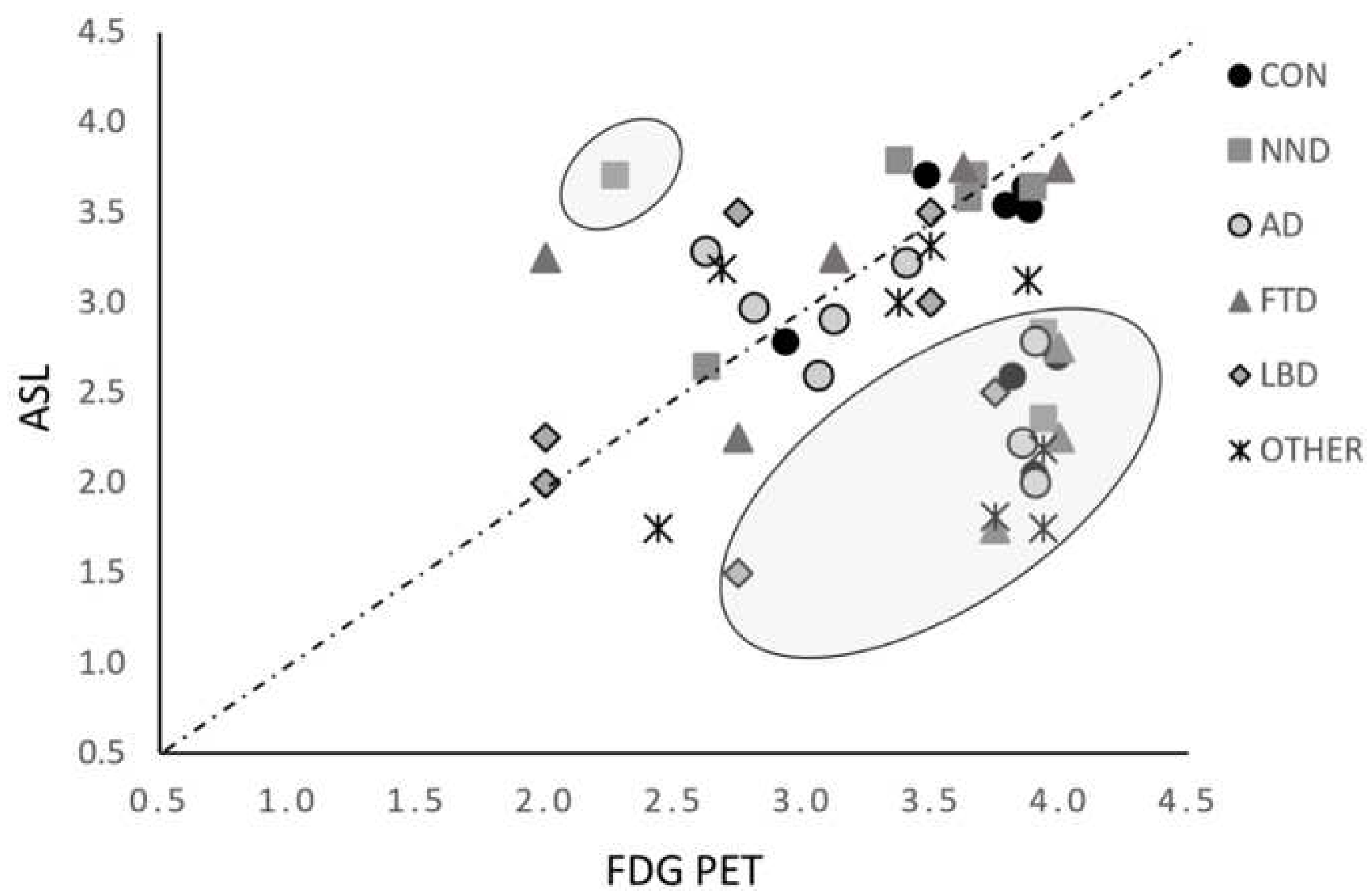


Figure 3

Click here to access/download;Figure;Fig.3.tif $\underline{\underline{\underline{*}}}$

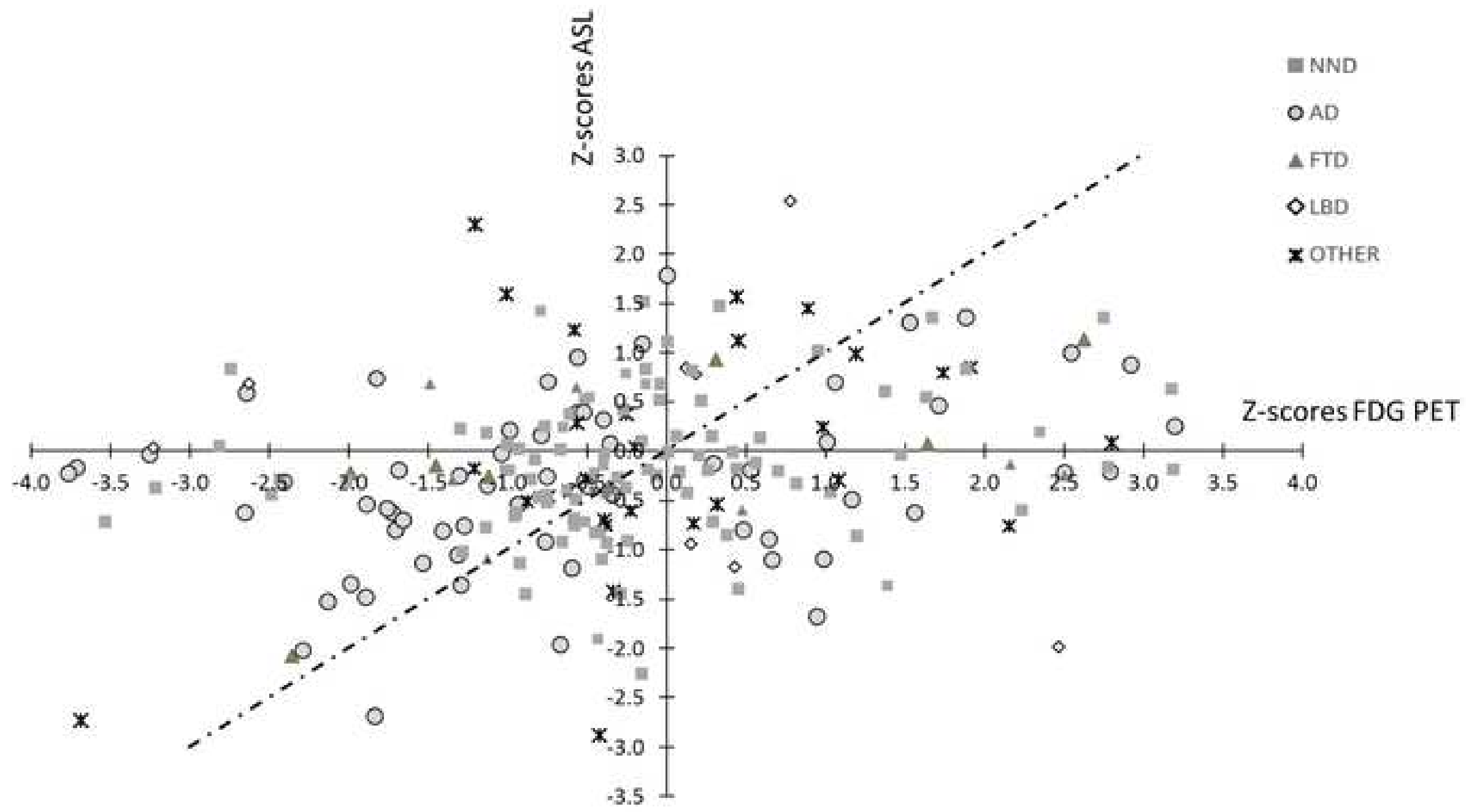


a) $\mathrm{AD}<\mathrm{CON}$

FDG

ASL

ASL $>$ FDG
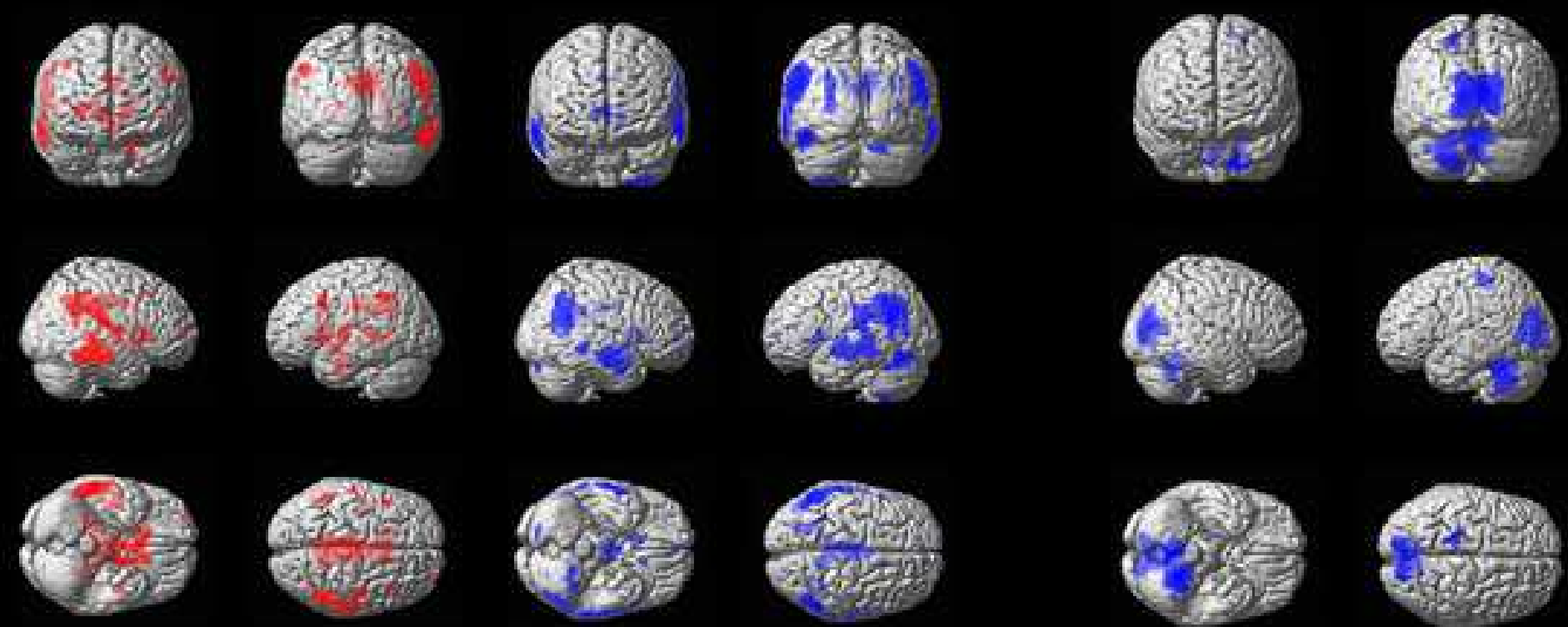

b) $\mathrm{FTD}<\mathrm{CON}$

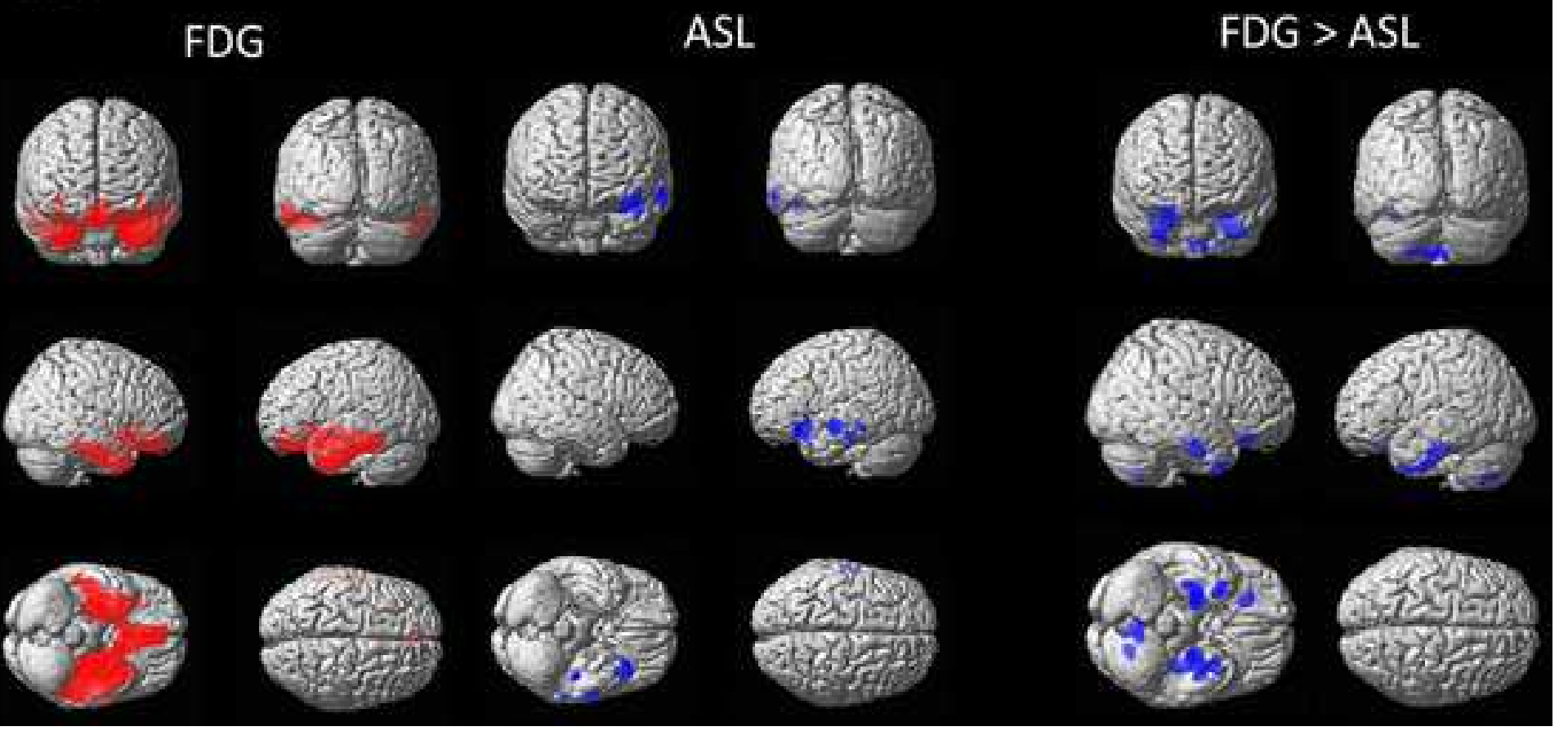




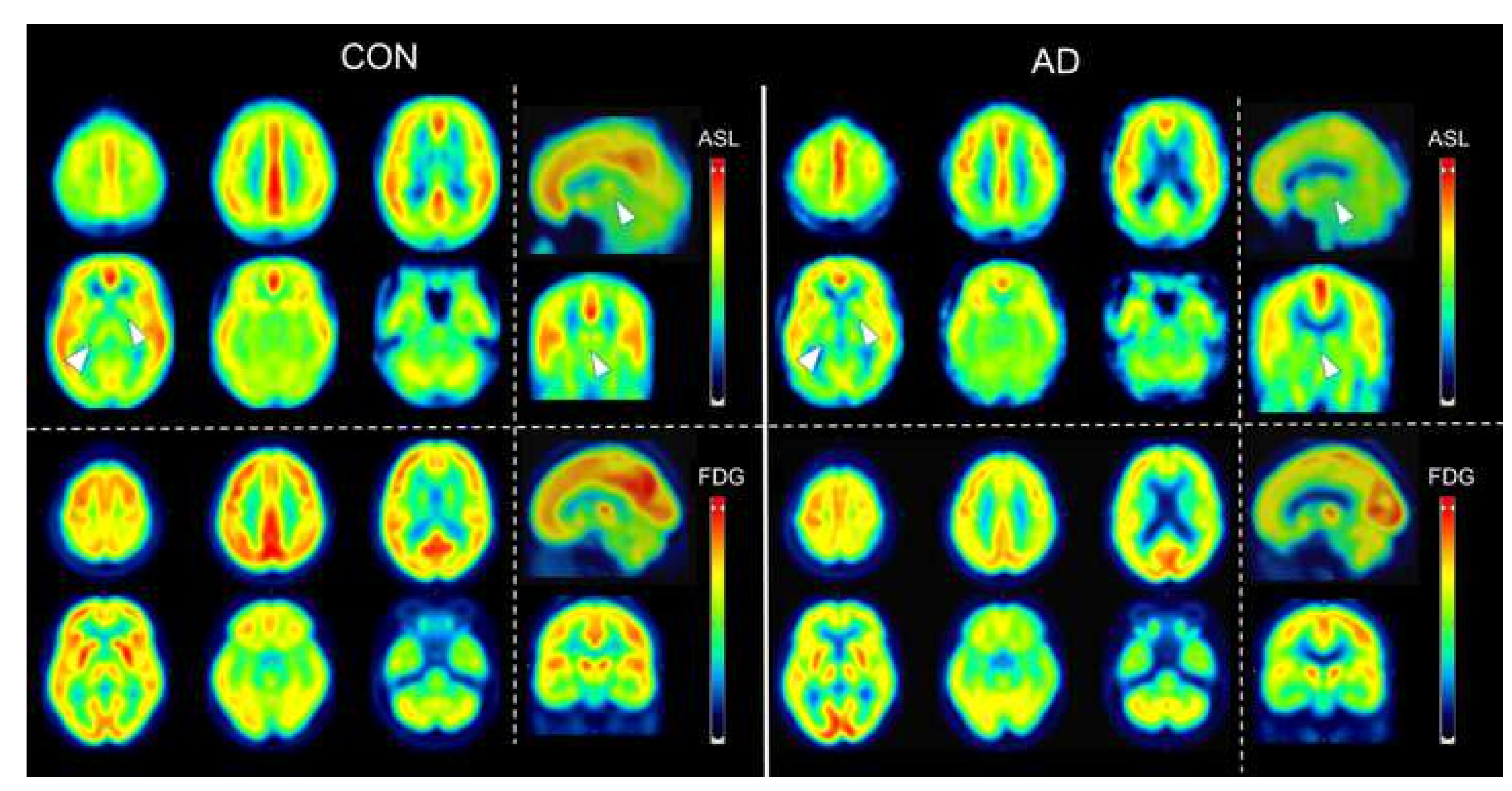


Click here to access/download Supplementary Material FDG PET vs ASL in dementia - PETMR study_Ceccarini_Sup Mat.docx 